\title{
The long-term impact of early treatment of multiple sclerosis on the risk of disability pension
}

\author{
Erik Landfeldt $^{1,2}$ (D) Anna Castelo-Branco ${ }^{1} \cdot$ Axel Svedbom $^{1} \cdot$ Emil Löfroth $^{3} \cdot$ Andrius Kavaliunas $^{4} \cdot$ Jan Hillert $^{4}$
}

Received: 22 November 2017 / Revised: 12 January 2018 / Accepted: 23 January 2018 / Published online: 1 February 2018

(c) The Author(s) 2018. This article is an open access publication

\begin{abstract}
Objective The objective of this retrospective, observational study was to estimate the long-term impact of early treatment of multiple sclerosis (MS) on the risk of disability pension.

Methods Our cohort comprised patients with MS in Sweden, identified in a nationwide disease-specific register (the Swedish Multiple Sclerosis Registry), who started treatment with a disease-modifying drug (DMD) between January 1, 2002, and December 31, 2012. We analyzed the association between time from onset of MS to treatment initiation and full-time disability pension using survival analysis.

Results Our sample comprised 2477 patients. Unadjusted Kaplan-Meier failure functions showed that patients who started treatment within six months after onset had a lower risk of disability pension across follow-up compared with patients initiating therapy after 12 months. Outcomes from the univariate Cox proportional hazards model showed that time from onset to treatment initiation (in years) was significantly associated with disability pension (HR 1.03, $p<0.001$ ). Outcomes from the multivariable Cox proportional hazards model showed that patients who started treatment within 6 months after onset had, on average, a $36 \%$ lower risk (HR $0.74, p=0.010$ ) of full-time disability pension during follow-up compared with patients starting treatment after 18 months when controlling for age, sex, marital status, university education, and prevalent comorbidities.

Conclusions We show that early treatment with DMDs of MS is associated with a significantly reduced risk of disability pension. Our findings highlight the potential long-term benefits of early treatment of MS and should be helpful to inform ongoing discussion on the optimum medical management of the disease.
\end{abstract}

Keywords Multiple sclerosis $\cdot$ Disability pension $\cdot$ Absenteeism $\cdot$ Social insurance $\cdot$ Survival analysis

Electronic supplementary material The online version of this article (https://doi.org/10.1007/s00415-018-8764-4) contains supplementary material, which is available to authorized users.

Erik Landfeldt

erik.landfeldt@ki.se

1 Mapi Group, Stockholm, Sweden

2 Institute of Environmental Medicine, Karolinska Institutet, Nobels väg 13, 17177 Stockholm, Sweden

3 Novartis, Stockholm, Sweden

4 Department of Clinical Neuroscience, Karolinska Institutet, Stockholm, Sweden

\section{Introduction}

In recent years, an extensive body of literature has accumulated with respect to benefits of early treatment of multiple sclerosis (MS) with disease modifying drugs (DMDs). Specifically, research has shown that early treatment in relation to disease onset is associated with significantly improved physical and mental outcomes, including lower relapse rates and lower Expanded Disability Status Scale (EDSS) scores, both in the short- and long-term [1-8]. In accordance with these findings, in several jurisdictions, including the UK [9] and Sweden [10], clinical consensus is now that DMDs should be offered as early as possible in the medical management of MS. However, there is still an ongoing discussion of the timing of treatment initiation [11], in part due to inconclusive results from observational effectiveness studies. 
With respect to the benefits of early treatment outside of the clinical trials setting, little is known of the long-term impact on socio-economic outcomes, including labour-force absenteeism. In our previous research, we have shown that patients with MS have elevated levels of sick leave and disability pension up to 15 years before disease diagnosis compared with the general population, highlighting the burden of disease on affected patients and society [12]. The objective of this study was to estimate the long-term impact of early treatment with DMDs on disability pension in patients with MS in Sweden.

\section{Methods}

\section{Study design and population}

This was a retrospective, observational study of the impact of early treatment on disability pension in patients with MS in Sweden. Our sample population was identified through a nation-wide disease-specific registry, the Swedish Multiple Sclerosis Registry [13] encompassing more than 70 care-units in regions across Sweden, and initially comprised all patients with MS that started treatment with a DMD [interferon beta (Avonex, Rebif, Betaferon, and Extavia), glatiramer acetate (Copaxone), natalizumab (Tysabri), fingolimod (Gilenya), and rituximab (Mabthera)] after January 1,2002 . We subsequently excluded patients younger than 18 years and older than 65 years at diagnosis (as they were not at risk for the study outcome, described below), patients with primary-progressive MS (due to the lack of standard treatment), and patients starting treatment after December 31, 2012 (to allow for a minimum of 1 year of follow-up and to avoid bias due to non-random censoring). Lastly, to facilitate interpretation of the analysis results (described below), patients were stratified into four pre-defined groups specified in terms of time from patient-reported onset of MS symptoms to initiation of first DMD treatment: (i) $<6$ months, (ii) 6-12 months, (iii) $12-18$ months, and (iv) $\geq 18$ months.

We extracted patient-level inpatient comorbidity data from the Swedish National Patient Register, data on age, sex, migration, marital status, and education level from the Swedish Total Population Register and the Longitudinal Integration Database for Health Insurance and Labor Market Studies (LISA), and dates of death from the Swedish Cause of Death Register.

\section{Outcomes}

The study outcome was defined as full-time (i.e., $100 \%$ of a full-time employment) disability pension. In Sweden, disability pension (i.e., sickness compensation, activity compensation, and early retirement pension) is granted to

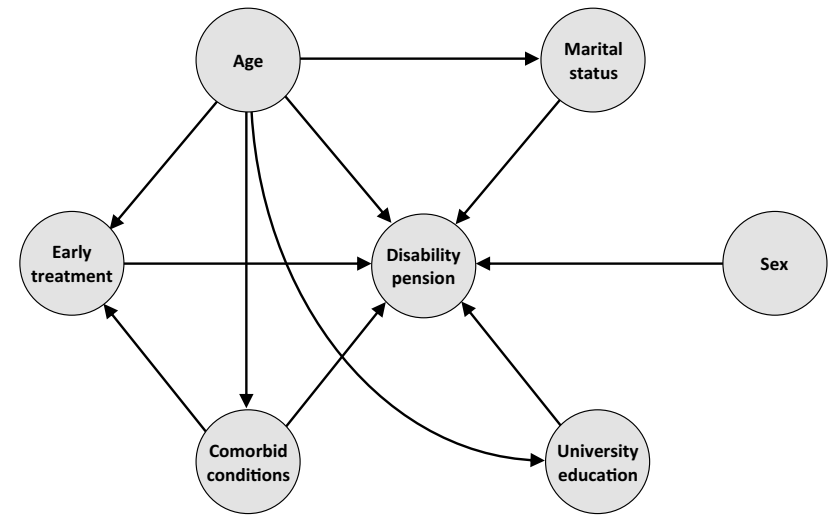

Fig. 1 Directed acyclic graph of candidate variables. Early treatment refers to timing of treatment initiation in relation to onset of MS symptoms. Age, university education, and marital status were measured at treatment initiation; comorbid conditions (primary and secondary diagnosis, as specified in the International Statistical Classification of Diseases and Related Health Problems) were measured 5 years preceding treatment initiation

individuals who are unlikely to return to full-time employment due to disease or injury. We analyzed the outcome using claims data from the Swedish Social Insurance Agency's database, Micro Data for Analysis of the Social Insurance (MiDAS) (data available up until December 31, 2013).

\section{Statistical analysis}

We investigated the association between early treatment of MS and full-time disability pension using survival analysis. Specifically, we first estimated Kaplan-Meier failure functions with time measured from treatment initiation to full-time disability pension (i.e., the failure event). In the analyses, patients were right-censored for emigration, age 65 years, death, and date of last available record from the registries (December 31, 2013).

Next, we used regression analysis to explore predictors and derive adjusted estimates of the association between early treatment of MS and full-time disability pension. Candidates for explanatory variables included age (at treatment initiation), sex, marital status (at treatment initiation), university education (at treatment initiation), and prevalent comorbidities (primary and secondary diagnosis, as specified in the International Statistical Classification of Diseases and Related Health Problems (ICD-10), 5 years prior to the start date of the DMD treatment). The causal structure of the dependent and independent variables was examined using a directed acyclic graph (DAG) (Fig. 1). A DAG is a didactic framework for modeling relationships and causality and can be utilized to help inform, e.g., model specification in regression analysis to reduce 
the degree of bias for the effect estimate [14-16]. In brief, in a DAG, nodes represent variables (e.g., age and sex) and directed (single-headed) arrows direct causal effects; a chain is given by $A \rightarrow D \rightarrow Y$ (i.e., $D$ mediates the effect of $A$ on $Y$ ), a fork is given by $A \leftarrow D \rightarrow Y$ (i.e., $D$ is a common cause of both $A$ and $Y$ ), and a collider (also known as inverted fork) is given by $A \rightarrow D \leftarrow Y$. An introduction to DAGs are provided by Fleischer et al. [17].

We hypothesized that age at treatment initiation was a common cause of early treatment (negative relationship, as younger patients on average would be more likely to have initiated treatment early in relation to onset of disease symptoms) and disability pension (positive relationship, as younger patients would have had symptoms for a shorter duration of time, which in turn may be associated with, e.g., a lower prevalence of MS-related comorbidities, as well as a relatively high propensity to seek healthcare in general), and comorbidities (within 5 years of treatment initiation) to be a common cause of early treatment (positive relationship, as those with many comorbid conditions would be expected to seek care and initiate treatment earlier) and disability pension (positive relationship, as comorbid conditions would be expected to be associated with a higher general risk of disability pension). As a consequence, to obtain meaningful estimates of the association between early treatment and disability pension (for which we designated a causal path), both of these confounding variables should be included in the multivariable regression analysis. We did not identify any colliders that would impact (i.e., induce conditional dependence) with respect to the association between the exposure variable (i.e., early treatment) and outcome variable (i.e., disability pension) and therefore decided to include all candidate variables in the final regression model (specified in detail below).

In total, we fitted two Cox proportional hazards models. The first model (Model A) was specified to include time (in years) from onset of MS to treatment as a continuous variable to investigate if a significant association was present. The second model (Model B) was specified to include time from onset of MS as a categorical variable (four strata, as defined above), as well as age (at treatment initiation), sex, marital status (at treatment initiation), university education (at treatment initiation), and prevalent comorbidities (as defined above) in accordance with our DAG analysis, with the aim to explore predictors and control for confounding effects. We tested the proportional hazards assumption on the basis of Schoenfeld residuals.

We compared demographic and disease characteristics across the four categories of time from onset of MS to treatment using Welch's analysis of variance and Pearson's Chi-square test. All analyses were conducted in Stata 14.

\section{Results}

A total of 2477 patients with MS initiated treatment with a DMD between January 1, 2002, and December 31, 2012 (maximum follow-up was 12 years; median 4 years). Table 1 presents summary characteristics of included patients, stratified by the four pre-defined strata of time from onset to treatment initiation. In the pooled sample, mean (SD) age at onset and diagnosis of MS was 33 (10) and 36 (10) years, respectively, 74\% (1845 of 2477) were female, $41 \%$ (1016 of 2477) were married, and $43 \%$ (1055 of 2477) had a university education at treatment initiation.

Figure 2 shows the unadjusted cumulative incidence proportion of full-time disability pension (100\% of a fulltime employment) after treatment initiation. Evident from the Kaplan-Meier failure functions, patients who started treatment within 6 months after onset (solid black line) had a markedly lower risk of disability pension across follow-up compared with patients initiating therapy after 12 months (dotted lines). Mean age at full-time disability pension was 40 years for patients who started treatment within 12 months, and 41 and 43 years for those who started within 12-18 months and after 18 months after disease onset, respectively. Complete life tables are available as supplemental material (Online Resource 1).

Results from the univariate Cox proportional hazards model (Model A) showed that time (in years) from onset of MS to treatment initiation was significantly associated with full-time disability (HR: 1.03, SD: 0.01, $p<0.001$ ). Results from the multivariable Cox proportional hazards model (Model B) are presented in Table 2. Patients who started treatment within 6 months after onset were estimated to have, on average, a $36 \%$ lower risk of permanently leaving the labor force to receive full-time disability pension compared with a patient starting treatment after 18 months when adjusting for age, sex, marital status, university education, and the prevalence of comorbidities. A similar trend, although not statistically significant, was noted for patients who started treatment 6-12 months after onset. As expected, we also found age, sex, and university education to be significant determinants of full-time disability pension.

\section{Discussion}

The objective of this study was to estimate the impact of early treatment on the risk of disability pension in patients with MS in Sweden. Our analyses show that early treatment with DMDs, as measured from onset of disease, was associated with a lower risk of full-time disability pension. Specifically, we found that patients who started treatment with a 
Table 1 Summary characteristics of included patients $(n=2477)$

\begin{tabular}{|c|c|c|c|c|c|}
\hline & \multicolumn{4}{|c|}{ Time from onset of MS to treatment initiation } & \multirow[t]{2}{*}{$p$ value } \\
\hline & $<6$ months & $6-12$ months & $12-18$ months & $\geq 18$ months & \\
\hline$n$ (proportion of total sample) & $704(28 \%)$ & $407(16 \%)$ & $190(8 \%)$ & $1176(47 \%)$ & \\
\hline Age, mean $(\mathrm{SD})$ years $^{\mathrm{a}}$ & $35(9.8)$ & $34.8(9.8)$ & $35.9(9.5)$ & $38.1(9.6)$ & $<0.001$ \\
\hline Sex, female & $892(76 \%)$ & $134(71 \%)$ & $316(78 \%)$ & $503(71 \%)$ & 0.041 \\
\hline Married $^{\mathrm{a}}$ & $233(33 \%)$ & $150(37 \%)$ & $76(40 \%)$ & $557(47 \%)$ & $<0.001$ \\
\hline University education $^{\mathrm{a}}$ & $290(41 \%)$ & $156(38 \%)$ & $87(46 \%)$ & $522(44 \%)$ & 0.115 \\
\hline Follow-up, mean (SD) months & $60.6(36.5)$ & $59.7(35.3)$ & $59.3(34.9)$ & $55.4(33.8)$ & 0.018 \\
\hline Time from onset to treatment initiation, mean (SD) months & $3.1(1.6)$ & $8.7(1.8)$ & $14.8(1.7)$ & $80.3(69.5)$ & $<0.001$ \\
\hline \multicolumn{6}{|l|}{ Prevalent comorbidities ${ }^{\mathrm{b}}$} \\
\hline Certain infectious and parasitic diseases & $28(4 \%)$ & $13(3 \%)$ & $7(4 \%)$ & $42(4 \%)$ & 0.924 \\
\hline Neoplasms & $15(2 \%)$ & $12(3 \%)$ & $3(2 \%)$ & $33(3 \%)$ & 0.616 \\
\hline $\begin{array}{l}\text { Diseases of the blood and blood-forming organs and certain disorders } \\
\text { involving the immune mechanism }\end{array}$ & $8(1 \%)$ & $4(1 \%)$ & $6(3 \%)$ & $9(1 \%)$ & 0.033 \\
\hline Endocrine, nutritional and metabolic diseases & $38(5 \%)$ & $26(6 \%)$ & $14(7 \%)$ & $58(5 \%)$ & 0.450 \\
\hline Mental and behavioural disorders & $26(4 \%)$ & $21(5 \%)$ & $12(6 \%)$ & $49(4 \%)$ & 0.358 \\
\hline Diseases of the nervous system ${ }^{c}$ & $225(32 \%)$ & $130(32 \%)$ & $39(21 \%)$ & $287(24 \%)$ & $<0.001$ \\
\hline Diseases of the eye and adnexa & $81(12 \%)$ & $44(11 \%)$ & $16(8 \%)$ & $90(8 \%)$ & 0.028 \\
\hline Diseases of the ear and mastoid process & $12(2 \%)$ & $5(1 \%)$ & $4(2 \%)$ & $33(3 \%)$ & 0.200 \\
\hline Diseases of the circulatory system & $40(6 \%)$ & $18(4 \%)$ & $10(5 \%)$ & $60(5 \%)$ & 0.836 \\
\hline Diseases of the respiratory system & $20(3 \%)$ & $22(5 \%)$ & $8(4 \%)$ & $38(3 \%)$ & 0.126 \\
\hline Diseases of the digestive system & $44(6 \%)$ & $29(7 \%)$ & $11(6 \%)$ & $61(5 \%)$ & 0.504 \\
\hline Diseases of the skin and subcutaneous tissue & $2(0 \%)$ & $4(1 \%)$ & $2(1 \%)$ & $11(1 \%)$ & 0.384 \\
\hline Diseases of the musculoskeletal system and connective tissue & $33(5 \%)$ & $22(5 \%)$ & $11(6 \%)$ & $77(7 \%)$ & 0.404 \\
\hline Diseases of the genitourinary system & $32(5 \%)$ & $21(5 \%)$ & $9(5 \%)$ & $62(5 \%)$ & 0.910 \\
\hline $\begin{array}{l}\text { Symptoms, signs and abnormal clinical and laboratory findings, not } \\
\text { elsewhere classified }\end{array}$ & $157(22 \%)$ & $100(25 \%)$ & $44(23 \%)$ & $232(20 \%)$ & 0.169 \\
\hline Injury, poisoning and certain other consequences of external causes & $28(4 \%)$ & $28(7 \%)$ & $10(5 \%)$ & $87(7 \%)$ & 0.023 \\
\hline
\end{tabular}

Data presented as $n(\%)$ if not specified otherwise

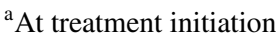

${ }^{\mathrm{b}}$ Includes primary and secondary inpatient care diagnosis within 5 years of treatment initiation

${ }^{\mathrm{c}}$ Excluding diagnosis of multiple sclerosis

DMD within 6 months after disease onset on average had a $36 \%$ lower risk of full-time disability pension during followup treatment compared with patient starting treatment after 18 months. These findings highlight the potential benefits of early treatment of MS and should be helpful to inform ongoing discussion on the optimum medical management of the disease.

Comparing our results with previous research, several clinical trials have demonstrated a positive effect of early treatment of MS. For example, Goodin et al. [1, 2] found that interferon beta treatment at onset was associated with better physical and mental outcomes, as well as reduced mortality, compared with those receiving treatment after 3 years of placebo. Rovaris et al. [3] found similar evidence for patients treated with glatiramer acetate, Cocco et al. [4] for patients treated with DMDs, and the PRISMS Study Group and the University of British Columbia MS/MRI Analysis Group
[5], Jacobs et al. [6], and Kappos et al. [7] for patients treated with interferon beta. More recently, in an observational study of Swedish patients with MS, Kavaliunas et al. [8] reported that patients had a $7 \%$ increased risk of reaching irreversible EDSS 4 for every year of delay in treatment start after MS onset. In this context, our study provides additional evidence that early treatment of MS may have a significant positive effect as studied outside the randomized controlled trial setting. Yet, additional research is needed to better understand the timing and impact of DMDs in the real-world clinical care of MS, in particular in the long term.

It should be noted that the association between early treatment and disability pension identified in our study may be subject to a phenomenon referred to by epidemiologists as confounding by indication. This bias arises when exposure (e.g., time from onset to treatment with DMDs) is associated (as a cause) with one or more patient characteristics 
Fig. 2 Cumulative incidence proportion of full-time disability pension
Table 2 Determinants of fulltime disability pension (Cox proportional hazards model, Model B)

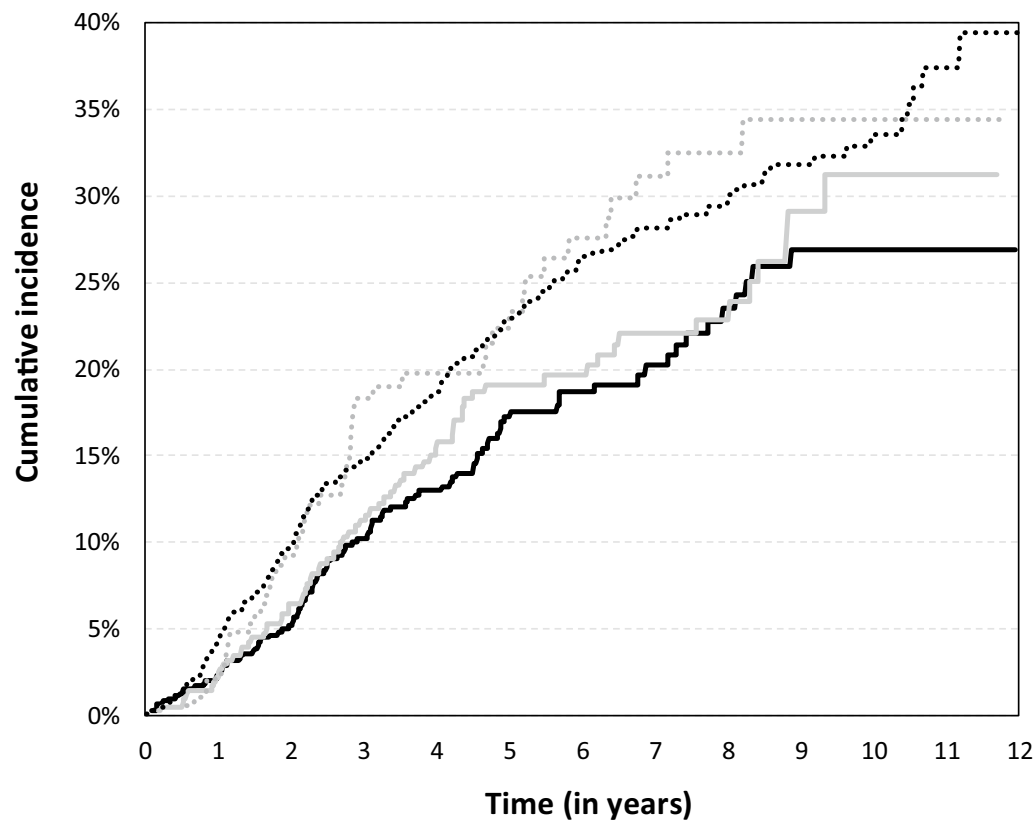

Number at risk

Time to treatment initiation

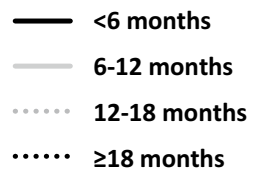

$\begin{array}{lllllllllllll}\mathbf{0} & \mathbf{1} & \mathbf{2} & \mathbf{3} & \mathbf{4} & \mathbf{5} & \mathbf{6} & \mathbf{7} & \mathbf{8} & \mathbf{9} & \mathbf{1 0} & \mathbf{1 1} & \mathbf{1 2} \\ 704 & 688 & 577 & 452 & 354 & 265 & 192 & 145 & 100 & 68 & 46 & 23 & 0 \\ 407 & 399 & 328 & 278 & 223 & 180 & 140 & 109 & 75 & 44 & 22 & 12 & 0 \\ 190 & 187 & 160 & 127 & 101 & 81 & 66 & 53 & 39 & 26 & 12 & 6 & 0 \\ 1176 & 1125 & 944 & 802 & 673 & 545 & 405 & 309 & 230 & 150 & 97 & 36 & 0\end{array}$

\begin{tabular}{lllrr}
\hline & Hazard ratio & Standard error & $p$ value & 95\% CI \\
\hline $\begin{array}{l}\text { Time from onset to treatment initiation } \\
\geq 18 \text { months (reference) }\end{array}$ & 1 & & & \\
12-18 months & 1.11 & 0.18 & 0.521 & $0.81-1.52$ \\
$6-12$ months & 0.78 & 0.11 & 0.074 & $0.59-1.02$ \\
$<6$ months & 0.74 & 0.09 & 0.010 & $0.58-0.93$ \\
Age, in years & 1.03 & 0.01 & $<0.001$ & $1.02-1.05$ \\
Sex, female & 1.34 & 0.15 & 0.009 & $1.08-1.67$ \\
Married & 0.91 & 0.09 & 0.342 & $0.74-1.11$ \\
University education & 0.53 & 0.05 & $<0.001$ & $0.44-0.64$ \\
\hline
\end{tabular}

Confidence interval (CI). Total sample: $n=2477$. Age, marital status, and university education were measured at treatment initiation. Robust estimates were obtained using the Lin and Wei estimator of variance. The model was also adjusted for the prevalence of comorbidity diagnosis groups (as listed in Table 1) 5 years preceding treatment initiation. The proportional hazards assumption was tested on the basis of Schoenfeld residuals (global test $p$ value $>0.05$ ) (e.g., age) that in turn are associated (as a cause) with the outcome of interest (e.g., disability pension). Specifically, in our study, patients who initiated treatment early may have had more pronounced manifestations of disease activity, and thus been subject to a more aggressive disease compared with patients starting treatment later after onset. These differences would imply that patients who received treatment early would be expected to have less favourable health outcomes in the long term. Indeed, we found evidence for this in our data when comparing comorbidities between the four treatment groups, where, e.g., those who started treatment early ( $<6$ months) had significantly higher prevalence of diseases of the nervous system compared with patients who started treatment after 18 months (Table 1). A similar pattern would be expected to also be applicable to patients starting treatment 18 months after onset as compared to those who start later (after, e.g., 36 months). Therefore, with respect to this potential bias, our estimate of a $36 \%$ risk reduction 
of full-time disability pension should be interpreted as conservative. Moreover, in our analysis, we did not account for differences in treatment algorithms of MS over time in Sweden or compliance to treatment, both which may have influenced our estimates. This would be an interesting topic for future research to help obtain further insight of the association between early treatment and socio-economic outcomes, such as disability pension, for this disease population.

In agreement with previous research [12, 18-20], we found that patients with a university education had a significantly lower probability of full-time disability pension. A possible explanation for this finding is that patients without a university degree to a larger extent may be employed to perform blue-collar work characterized by, e.g., heavy lifts and strict work hours, which may be more difficult to maintain for a patient with MS. Another explanation could be that patients with a university education are more likely to seek healthcare and thus maintain healthy also in more advanced ages, a pattern identified in previous research of, e.g., myocardial infarction [21]. We also found age to be associated with an increased risk of disability pension, which would be expected to be a function of the natural evolution of MS, with increased disability and morbidity in higher ages, as well as the elevated morbidity profile of the elderly general population. Moreover, females were found to have a significantly higher risk of disability pension compared to men, a pattern also prevalent in the general Swedish population [22]. Possible reasons for this finding include gender segregation in the labour market in Sweden (where women to a larger extent than men have monotonous occupations that involve a high degree of repetitive strain with lower salary and limited involvement in decision-making) and fact that women on average carry a much larger burden of the work at home, which has been shown to be associated with impaired health [23].

A limitation of the present study concerns our inability to control for EDSS at treatment initiation due to lack of data. However, in a study of early treatment with DMDs on EDSS by Kavaliunas et al. [8], baseline EDSS was found to be similar for patients starting treatment within 1 year (mean: 1.6) and one to 3 years (mean: 1.6) after disease onset. This indicates that the impact of omitting this variable in our analysis may have been minor. Moreover, we did adjust the multivariable regression model for age, which we previously have shown to be a relatively robust proxy for disease progression in MS when analyzing medical absenteeism (i.e., sick leave and disability pension) [12]. A second limitation concerns our comorbidity data, which only comprised inpatient diagnoses. Consequently, the absolute levels of the reported conditions should be interpreted with caution. A third limitation concerns the retrospective nature of our data, which implies that we were unable to infer causality for the identified associations. Strengths of the present work include the relatively large sample of patients, the robust longitudinal datasets comprising both long-term clinical data from the Swedish Multiple Sclerosis Registry and demographic variables from several administrative databases in Sweden (covering the total Swedish population) which also allowed censoring for, e.g., death and emigration in our survival analysis.

In conclusion, we show that early treatment with DMDs of MS is associated with a significantly reduced risk of disability pension. These findings should help inform therapeutic decisions in MS. Yet, more research is needed to fully understand the timing of treatment with DMDs on long-term health and socio-economic outcomes in MS.

Acknowledgements This study was funded by Novartis.

\section{Compliance with ethical standards}

Conflicts of interest Dr. Erik Landfeldt, Dr. Anna Castelo-Branco, and Mr. Axel Svedbom are consultants to Novartis through their employment at Mapi Group. Mr. Emil Löfroth is an employee and shareholder of Novartis. Dr. Andrius Kavaliunas reports no conflicts of interest. Dr. Jan Hillert has received honoraria for serving on advisory boards for BiogenIdec and Novartis, speaker's fees from BiogenIdec, MerckSerono, Bayer-Schering, Teva, and Sanofi-Aventis, and has served as investigator for projects sponsored by, or received unrestricted research support from, BiogenIdec, Merck-Serono, TEVA, Novartis, and BayerSchering.

Ethical approval The study ethical approval was granted by the Regional Ethical Review Board in Stockholm, Sweden.

Informed consent Patient identifiers were encrypted.

Open Access This article is distributed under the terms of the Creative Commons Attribution 4.0 International License (http://creativeco mmons.org/licenses/by/4.0/), which permits unrestricted use, distribution, and reproduction in any medium, provided you give appropriate credit to the original author(s) and the source, provide a link to the Creative Commons license, and indicate if changes were made.

\section{References}

1. Goodin DS, Traboulsee A, Knappertz V et al (2012) Relationship between early clinical characteristics and long term disability outcomes: 16 year cohort study (follow-up) of the pivotal interferon B-1b trial in multiple sclerosis. J Neurol Neurosurg Psychiatry 83(3):282-287

2. Goodin DS, Ebers GC, Cutter G et al (2012) Cause of death in MS: long-term follow-up of a randomised cohort, 21 years after the start of the pivotal IFN -1b study. BMJ Open 2(6):001972

3. Rovaris M, Comi G, Rocca MA et al (2007) Long-term follow-up of patients treated with glatiramer acetate: a multicentre, multinational extension of the European/Canadian double-blind, placebocontrolled, MRI-monitored trial. Mult Scler 13(4):502-508

4. Cocco E, Sardu C, Spinicci G et al (2015) Influence of treatments in multiple sclerosis disability: a cohort study. Mult Scler 21(4):433-441 
5. PRISMS Study Group and the University of British, Columbia MS/MRI Analysis Group (2001) PRISMS-4: long-term efficacy of interferon-beta-1a in relapsing MS. Neurology 56(12):1628-1636

6. Kappos L (2004) Effect of drugs in secondary disease progression in patients with multiple sclerosis. Mult Scler 10(Suppl 1):S46-S54

7. Jacobs LD, Beck RW, Simon JH et al (2000) Intramuscular interferon beta-1a therapy initiated during a first demyelinating event in multiple sclerosis. N Engl J Med 343:898-904

8. Kavaliunas A, Manouchehrinia A, Stawiarz L et al (2017) Importance of early treatment initiation in the clinical course of multiple sclerosis. Mult Scler 23(9):1233-1240

9. Multiple Sclerosis Society. Time to Act-a consensus on early treatment. http://mssociety.org.uk/earlytreatment. Accessed 10 Aug 2017

10. The National Board of Health and Welfare (Socialstyrelsen) (2017) Vård vid multipel skleros och Parkinsons sjukdom. Nationella riktlinjer. http://www.socialstyrelsen.se/nationellariktl injermsochparkinsonssjukdom. Accessed 15 Sep 2017

11. Gilmore CP, Cottrell DA, Scolding NJ et al (2010) A window of opportunity for no treatment in early multiple sclerosis? Mult Scler 16:756-759

12. Landfeldt E, Castelo-Branco A, Svedbom A et al (2016) Sick leave and disability pension before and after diagnosis of multiple sclerosis. Mult Scler 22(14):1859-1866

13. Hillert J, Stawiarz L (2015) The Swedish MS registry-clinical support tool and scientific resource. Acta Neurol Scand 132(199):11-19

14. Pearl J (1995) Causal diagrams for empirical research. Biometrika 82:669-710
15. Greenland S, Pearl J, Robins JM (1999) Causal diagrams for epidemiologic research. Epidemiology 10:37-48

16. Shrier I, Platt RW (2008) Reducing bias through directed acyclic graphs. BMC Med Res Methodol 8:70

17. Fleischer NL, Diez Roux AV (2008) Using directed acyclic graphs to guide analyses of neighbourhood health effects: an introduction. J Epidemiol Community Health 62(9):842-846

18. Tinghög P, Hillert J, Kjeldgård L et al (2013) High prevalence of sickness absence and disability pension among multiple sclerosis patients: a nationwide population-based study. Mult Scler 19:1923-1930

19. Julian L, Vella L, Vollmer T et al (2008) Employment in multiple sclerosis. J Neurol 255:1354-1360

20. Patti F, Pozzilli C, Montanari E et al (2007) Effects of education level and employment status on HRQoL in early relapsingremitting multiple sclerosis. Mult Scler 13:783-791

21. Ringbäck Weitoft G, Ericsson O, Löfroth E, Rosén M (2008) Equal access to treatment? Population-based follow-up of drugs dispensed to patients after acute myocardial infarction in Sweden. Eur J Clin Pharmacol 64(4):417-424

22. Angelov N, Johansson P, Lindahl E, et al (2017) Kvinnors och mäns sjukfrånvaro. Rapport 2011:2. The Institute for Evaluation of Labour Market and Education Policy. http://www.ifau.se. Accessed 3 Sep 2017

23. Boye K (2008) Happy hour? Studies on well-being and time spent on paid and unpaid work. The Swedish Institute for Social Research dissertation series no. 74. Stockholm: Institutet för social forskning (SOFI) 